\title{
Review Article \\ PET/CT in the Staging of the Non-Small-Cell Lung Cancer
}

\author{
Fangfang Chao ${ }^{1,2,3,4}$ and Hong Zhang1, 2,3,4 \\ ${ }^{1}$ Department of Nuclear Medicine, Second Affiliated Hospital of Zhejiang University School of Medicine, Hangzhou, \\ Zhejiang 310009, China \\ ${ }^{2}$ Zhejiang University Medical PET Center, Zhejiang University, Hangzhou 310009, China \\ ${ }^{3}$ Institute of Nuclear Medicine and Molecular Imaging, Zhejiang University, Hangzhou 310009, China \\ ${ }^{4}$ Key Laboratory of Medical Molecular Imaging of Zhejiang Province, Hangzhou 310009, China
}

Correspondence should be addressed to Hong Zhang, hzhang21@gmail.com

Received 1 January 2012; Accepted 20 January 2012

Academic Editor: Mei Tian

Copyright (C) 2012 F. Chao and H. Zhang. This is an open access article distributed under the Creative Commons Attribution License, which permits unrestricted use, distribution, and reproduction in any medium, provided the original work is properly cited.

Lung cancer is a common disease and the leading cause of cancer-related death in many countries. Precise staging of patients with non-small-cell lung cancer plays an important role in determining treatment strategy and prognosis. Positron emission tomography/computed tomography (PET/CT), combining anatomic information of CT and metabolic information of PET, is emerging as a potential diagnosis and staging test in patients with non-small-cell lung cancer (NSCLC). The purpose of this paper is to discuss the value of integrated PET/CT in the staging of the non-small-cell lung cancer and its health economics.

\section{Introduction}

Lung cancer, with $80-85 \%$ being non-small-cell lung cancer (NSCLC), is the leading cause of cancer-related death in both men and women in the western world [1]. In 2008, 215,020 new cases are expected and 161,840 persons are projected to die from the disease in the United States [2]. Adequate therapeutic planning and prognosis is largely depended on the early diagnosis and precise staging. Computed tomography (CT) as a conditional and standard method of diagnosis and staging lung cancer provides excellent morphological information, but it has significant limitations in differentiating between benign and malignant lesions either in an organ or in lymph nodes [3]. Positron emission tomography (PET) is a unique imaging technique that provides details of functional processes in the body. However, the pool anatomic details obtained with PET make it hard to locate lesions, which can lead to errors in the diagnosis and staging of NSCLC. The combination of the 2 imaging procedures provided by integrated PET/CT scanners is relatively new, which was introduced into clinical practice in 1998 [4]. Advances in positron emission tomography combined with computed tomography (PET/CT) strive to solve these problems by allowing the acquisition of both functional and anatomical information of the whole body in a single study and has gained wide acceptance over the last few years, especially in North America and Western Europe, and its clinical utility is expected to continue to rise [5]. The purpose of this paper is to discuss the value of integrated PET/CT in the staging of the non-small-cell lung cancer and its health economics.

\section{Imaging Technique}

A very small amount of a biological compound labeled with a positron-emitting radionuclide, which is produced in a cyclotron and has a very short half live, is injected intravenously. A PET scanner measures the localization of the tracer in tissue. The most commonly used radionuclide is Fluoride $\left({ }^{18} \mathrm{~F}\right)$ for its relatively long half-life $(110 \mathrm{~min})$. Glucose which is usually used to provide energy for our body, is the most commonly used biologic agent. Glucose is labeled with ${ }^{18} \mathrm{~F}$ to create the glucose analog ${ }^{18} \mathrm{~F}$-fluorodeoxyglucose $\left({ }^{18} \mathrm{~F}-\mathrm{FDG}\right)$ that is the most widely used radionuclide in oncology because cancer cells have greater metabolic activity compared with normal cells. The radionuclides used in PET emit positrons as they decay. These positrons annihilate after encountering an electron and produce a pair of 
photons that travel in opposite directions, which are then detected by the PET scanner. The PET scan detects these annihilation photons and is able to construct tomography images including coronal, sagittal, and transverse manners, which embody quantitative physiological, pathological, or pharmacological information [6].

Cancer cells are capable of greater intracellular uptake of FDG because of increased glucose transporters on the cell membrane and increased activity of enzymes involved in the glycolytic pathway. FDG is phosphorylated to FDG6-phosphate which, cannot be further metabolized and remains trapped in the cells. Therefore, the uptake and accumulation of malignant regions will be greater than normal tissue cells and they will be displayed as abnormal strong gather areas.

Due to the relatively poor spatial resolution of PET, disease localization usually proved to be hard. To solve this problem, anatomic and functional imaging has been integrated into one diagnostic modality that is known as image fusion. Image fusion can be performed at 3 different levels [7]: visual fusion, software fusion, and hardware fusion. In traditional visual image fusion, the physician compares 2 separate imaging modalities viewed next to each other. The fusion takes place in his or her mind. In software image fusion, PET and CT imaging are fused using softwarebased algorithms, which creates $2 \mathrm{D}$ and 3D fusion images. As far as integrated PET/CT is considered, hardware fusion using a single detector provides the best coregistration of physiologic and anatomic detail.

Hardware fusion is the most advanced and can make best anatomic registration, but it is the most expensive and the least accessible method. In the integrated PET/CT study, low-dose CT, diagnostic CT and PET scans are all obtained in a single time. Low-dose CT obtained at quiet respiration for attenuation correction results in a lower noise emission scan and faster examinations (reduction of whole body scan times by at least $40 \%$ ) and, thus, fewer motion artifacts and a higher throughput [8]. The diagnostic CT, obtained with the administration of contrast material, provides excellent anatomic data. Fusion obtained using external software, which creates 2D and 3D fusion images [9].

\section{Staging}

Accurate staging of patients with non-small-cell lung cancer is critical in determining treatment strategy and predicting prognosis. Nowadays we usually take TNM staging system, which is maintained by the American Joint Committee on Cancer and the International Union Against Cancer [10]. TNM staging system is based on a combination of findings: the location and extent of the primary tumor $(\mathrm{T})$, the presence or absence of intrapulmonary, hilar or mediastinal lymph node metastases $(\mathrm{N})$, and the presence or absence of extrathoracic metastases (M) $[11,12]$. The combination of $\mathrm{T}, \mathrm{N}$, and $\mathrm{M}$ staging is then used to give the tumor an overall stage (I-IV), with the aim of grouping patients into stages with similar prognosis. Survival rate and treatment options also vary from stage to stage [10].
Based on adequate diagnosis and staging, personalized treatment strategies can be selected to gain best prognosis and to be cost-effective. The recommended therapy [13] for stage I disease is surgical resection, with stereotactic body radiation therapy (SBRT) $[14,15]$ reserved for those who are medically inoperable. Stage II disease is also treated with surgery followed by adjuvant chemotherapy to prevent disease recurrence. Stage IIIA disease has multiple treatment options determined by the extent of regional (nodal) involvement. Stage IIIA disease is often treated with concurrent chemotherapy and radiation, adding surgical resection (trimodality therapy) for those who are medically fit and have responded well to initial concurrent therapy. Stage IIIB disease is treated with concurrent chemotherapy and radiation. Stage IV disease is treated with systemic therapy, chemotherapy, and/or molecular targeted agents, in addition to radiotherapy for alleviating of painful lesions or brain metastasis. Many studies have demonstrated that integrated PET/CT is more accurate than CT alone, PET alone $[16,17]$, and visually correlated PET/CT in evaluating, the TNM status [18].

3.1. T Staging. $T$ staging describes the location, size, and extension of the primary tumor and the presence or absence of satellite nodules. CT is an important imaging modality for the evaluation of the primary tumor because of its excellent anatomical resolution. But it is difficult for it to evaluate the invasion of the chest wall or involvement of the mediastinum and differentiate tumors from postobstructive atelectasis correctly [19].

FDG-PET gives more information about the metabolic changes of the neoplasm but offers little application in the $\mathrm{T}$ staging of non-small-cell lung cancer, because of its limited ability for precise anatomic location and size measurement. PET is limited in detecting microscopic tumor deposits, correctly evaluating extension of tumor and biological low metabolism tumor, such as bronchoalveolar cell carcinoma, carcinoid tumors, and some adenomas [18]. It is striking that PET alone are both upstaging and understaging for the $\mathrm{T}$ staging of patients with NSCLC.

It has been shown that integrated PET/CT provided more than the sum of PET and CT [20]. In particular, it improves T staging [21]. Due to precise CT correlation with the extent of ${ }^{18} \mathrm{~F}-\mathrm{FDG}$ uptake, the location of the primary tumor can be exactly defined. It has been demonstrated that integrated PET/CT provides important information on mediastinal infiltration, chest wall infiltration, and differentiation between tumor and peritumoral atelectasis [3]. It has been found in many literatures [21] that PET/CT is the best noninvasive imaging technique for the accurate prediction of T staging. In one study of Wever et al. [18], integrated $\mathrm{PET} / \mathrm{CT}$ correctly predicted the $\mathrm{T}$ staging in patients with NSCLC in $86 \%$ of cases versus $68 \%$ with CT, $46 \%$ with PET, $72 \%$ with visually correlated PET/CT.

3.2. N Staging. Accurate mediastinal staging is particularly important, as in many cases the status of these nodes will determine whether surgical resection of lung cancer is 
TABLE 1: Recent studies evaluating N staging with PET/CT compared with PET and CT.

\begin{tabular}{|c|c|c|c|c|c|c|c|c|c|c|c|c|c|c|c|}
\hline \multirow{2}{*}{ Ref. } & \multicolumn{3}{|c|}{ Sensitivity (\%) } & \multicolumn{3}{|c|}{ Specificity (\%) } & \multicolumn{3}{|c|}{ PPV (\%) } & \multicolumn{3}{|c|}{ NPV (\%) } & \multicolumn{3}{|c|}{ Accuracy (\%) } \\
\hline & $\mathrm{PET} / \mathrm{CT}$ & PET & CT & $\mathrm{PET} / \mathrm{CT}$ & PET & CT & $\mathrm{PET} / \mathrm{CT}$ & PET & CT & $\mathrm{PET} / \mathrm{CT}$ & PET & CT & $\mathrm{PET} / \mathrm{CT}$ & PET & CT \\
\hline [25] & 70 & & & 94 & & & 64 & & & 95 & & & & & \\
\hline [26] & & & & 95 & & 77 & & & & & & & 87 & & 69 \\
\hline [27] & 54 & & & 92 & & & 74 & & & 82 & & & 81 & & \\
\hline [28] & 65 & & & 97 & & & 79 & & & 90 & & & 92 & & \\
\hline [29] & 86 & & 69 & 85 & & 71 & 64 & & 43 & 95 & & 88 & 85 & & 71 \\
\hline [18] & 83 & 83 & 83 & 84 & 81 & 68 & 75 & 71 & 60 & 90 & 89 & 88 & 84 & 82 & 74 \\
\hline [30] & 84 & & 84 & 85 & & 61 & & & & & & & 85 & & 74 \\
\hline [31] & 56 & & 65 & 100 & & 89 & & & & & & & 90 & & 83 \\
\hline$[20]$ & 85 & & 70 & 84 & & 69 & & & & & & & 84 & & 69 \\
\hline Average & 73 & 83 & 74 & 91 & 81 & 73 & 71 & 71 & 52 & 90 & 89 & 88 & 86 & 82 & 73 \\
\hline
\end{tabular}

possible [3]. The accuracy of CT for determining $\mathrm{N}$ staging remains limited, because nodal staging with CT is based on morphological characteristics. Lymph node size is used as the only criterion to determine metastatic disease. The current consensus considers a lymph node with a shortaxis diameter greater than $1 \mathrm{~cm}$ as a predictor for metastasis [22]. However, if postobstructive pneumonitis is present, little correlation exists between the size of the mediastinal lymph nodes and tumor involvement [23]. Normal-sized regional lymph nodes may prove to be metastasizing upon histological examination, and nodal enlargement can be due to reactive hyperplasia or other nonmalignant conditions. PET has been reported to increase diagnostic accuracy in the differentiation of benign and malignant lesions and to improve identification of nodal metastasis. Functional scans obtained with FDG PET not only are complementary to those obtained with conventional modalities but also may be more sensitive because alterations in tissue metabolism generally precede anatomic change [24]. However, its poor spatial detail and FDG being nonspecific tracer can lead to inaccuracies, particularly in the areas of normal physiologic uptake.

By integrating functional and anatomic data, PET/CT improved N staging compared with PET or CT alone. Indeed the benefit of PET/CT compared with PET in nodal staging appears to lie in a moderate increase in specificity and positive predictive value for its additional exactly anatomic information. Because of attaching metabolism information of lymph node, PET/CT improves accuracy compared with CT. Initial studies demonstrated a pooled average sensitivity, specificity, positive predictive value, negative predictive value, and accuracy of PET/CT for detecting metastatic lymph nodes of, respectively, $73 \%, 91 \%, 71 \%, 90 \%$, and $86 \%$ versus $83 \%, 81 \%, 71 \%, 89 \%, 82 \%$ of PET alone and $74 \%$, $73 \%, 52 \%, 88 \%, 73 \%$ of CT alone. The results of these studies are summarised in Table $1[18,20,25-31]$.

In one study performed by Darling et al. [25], of 22 patients with a PET/CT interpreted as positive for mediastinal nodes, 8 did not have tumor. Based on PET/CT alone, eight patients would have been denied potentially curative surgery if the mediastinal abnormalities detected by PET/CT had not been evaluated with an invasive mediastinal procedure. PET/CT assessment of the mediastinum is associated with a clinically relevant false-positive rate. When positive mediastinal lymph nodes are detected, invasive mediastinal staging must be performed [25]. While mediastinal lymph nodes is negative, surgery can be done directly because of its high negative predict value.

There is an ongoing controversy whether PET/CT scan can reduce further invasive mediastinal staging. The general consensus is that PET/CT can reduce mediastinoscopy for high negative predict value. Perigaud et al. [32] reported the specificity is high: patients with negative integrated ${ }^{18}$ F-FDG PET/CT can be operated upon directly without invasive mediastinal staging. While one study of Metin et al. [33] showed that PET/CT does not reduce the need for invasive procedures in detecting lymph node metastasis in aortopulmonary window. By integrating functional and anatomic data, PET/CT is the best noninvasive method for the detection of nodal metastasis, but mediastinoscopy remains the gold standard $[25,33]$. The results of imaging are not conclusive and the probability of mediastinal involvement is high (based on tumor size and location), which are the indications for mediastinoscopy. Therefore, mediastinoscopy is appropriate for patients with T2 and T3 lesions even if the PET/CT scan does not suggest mediastinal node involvement. Mediastinoscopy may also be appropriate to confirm mediastinal node involvement in patients with a positive PET/CT scan [12]. In general, the biopsy of the lymph node with the highest stage by endoscopic bronchial ultrasound (EBUS) or mediastinoscopy is sufficient for further treatment decisions. However, not all mediastinal lymph nodes are routinely reachable by EBUS or mediastinoscopy (paraaortic region, aortopulmonary window). Recently, it has been demonstrated that PET can assist mediastinoscopy [3].

3.3. M Staging. Despite radical surgical treatment of potentially curable NSCLC the overall 5-year survival rate remains low $(20-40 \%)$. One reason for this is undetected extrathoracic metastases, which cause underestimation of the tumor 
stage [3]. NSCLC most metastasizes to the brain, bones, liver, and adrenal glands [34].

Radionuclide bone scanning with ${ }^{99 \mathrm{~m}} \mathrm{Tc}$-methylene diphosphonate is usually used in the detection of occult skeletal metastases; however, it has high false-positive rate [35]. The accuracy of bone scintigraphy to detect bone metastases was $87 \%$ versus $98 \%$ of ${ }^{18} \mathrm{~F}-\mathrm{FDG}$ PET. PET scanning is more sensitive and accurate than bone scanning for the detection of skeletal metastases $(91 \%$ and $94 \%$ versus $75 \%$ and $85 \%$, resp.), with a high PPV of $98 \%$ if the findings on PET/CT scanning are concordant, but decreases to $61 \%$ if the CT scan is negative [36].

Adrenal metastases from lung cancer are common, found in approximately $33 \%$ of patients at autopsy [12]. In patients with NSCLC, however, many solitary adrenal masses are not malignant. So it is very critical in distinguishing between a metastatic lesion and an adenoma. Diagnostic CT evaluates adrenal metastases based on tumor size and attenuation value, which may lead to false negative or false positive results. Any adrenal mass found on a preoperative CT scan in a patient with lung cancer should be biopsied to rule out benign adenoma. For magnetic resonance imaging (MRI), the ability to distinguish metastases from adenomas has improved considerably with the introduction of more sophisticated MR imaging techniques such as fatsaturated, chemical shift and dynamic gadolinium-enhanced MR imaging. One group of investigators correlated the MR imaging findings with histological results in 114 patients with 134 adrenal masses. Combined chemical shift and dynamic gadolinium-enhanced MR imaging was found to have a sensitivity of $91 \%$ and a specificity of $94 \%$ for differentiating benign from malignant adrenal masses [37]. In fact, PET scanning is useful in distinguishing benign from malignant adrenal masses detected on CT scanning [35, 38]. In some studies, integrated PET/CT is a useful diagnostic modality for adrenal gland imaging in cancer patients, since it allows early detection and accurate localization of adrenal lesions and differentiation of metastatic nodules from benign lesions, thereby facilitating treatment planning $[39,40]$. However, some adenoma may show increased FDG, which will produce false positive results. Hemorrhage, necrosis [37, 41], and subcentimeter metastatic lesions may have low FDG uptake, which will lead to produce false negative results. In one study performed by Perri et al. [42], they demonstrated that the combination of standardized uptake value (SUV) and CT histogram analysis allowed us to significantly improve the PET/CT diagnostic accuracy for characterizing adrenal lesions, leading to a significant reduction in the number of false positive cases. In another study, Chong et al. [43] reported that fine-needle aspiration biopsy should be performed only if clinical and imaging studies are inconclusive.

In the newly adopted 7th edition of the TNM classification for lung cancer, NSCLC metastatic is subdivided into M1a for those cases with pleural nodules or malignant pleural or pericardial effusion and additional pulmonary nodules in the contralateral lung and M1b for those cases with other distant metastases disease [44]. The presence of pleural or pericardial nodules on CT can confirm the diagnosis of M1a disease, but these findings are often absent. PET can usually suggest the diagnosis of malignant pleural disease by showing increased focal or diffuse FDG uptake, but localization to the pleura or pericardium is not always clear for its poor resolution. Statistically, PET/CT has proven to be superior to either technique the in evaluation of metastatic pleural disease.

PET/CT has a low sensitivity in detecting brain metastases because of brain cells with high glucose uptake in nature. Therefore, in patients with neurological symptoms, an MRI of the brain should be performed, because of its high resolution, while Salskov et al. reported that PET using ${ }^{18} \mathrm{~F}$ fluorothymidine $\left({ }^{18} \mathrm{~F}-\mathrm{FLT}\right)$ for its low physiological uptake in brain can be used in detecting brain metastases [45].

PET/CT can improve the accuracy in the staging of non-small-cell lung cancer. For N staging, PET/CT scanning possessing high negative predict value can reduce unnecessary mediastinoscopy. Although some authors found no significant reduction in the number of avoidable thoracotomies performed when employing additional PET imaging in NSCLC, the general consensus is that PET can reduce needless thoracotomy rates. In one study of Fischer et al. [46], a total of 189 patients were enrolled and randomly assigned to either the PET/CT group (98 patients) or the conventional-staging group (91 patients). After staging, 60 patients in the PET/CT group (61\%) and 73 patients in the conventional-staging group (80\%) were considered to have operable disease and underwent thoracotomy $(P=0.004)$, while a total of 21 of 98 patients in the PET/CT group (21\%) and 38 of 91 in the conventional-staging group (42\%) underwent a futile thoracotomy. In other words, for every five PET/CT scans, one futile thoracotomy was avoided. Therefore, they reported that the use of PET/CT for preoperative staging of NSCLC reduced both the total number of thoracotomies and the number of futile thoracotomies. Two randomized trials have assessed the clinical effect of PET alone. A trial by van Tinteren et al. [47] showed that the number of futile thoracotomies was significantly reduced in $19 / 82$ patients compared to $29 / 96$ patients, based on the addition of ${ }^{18} \mathrm{~F}$-FDG PET to the diagnostic algorithm. A randomized trial, however, did not show that adding PET reduced the number of thoracotomies [48]. We have to notice that imaging strategies based on PET/CT may help identify advanced disease and prevent futile thoracotomies in patients with NSCLC, but it also has false positive results that incorrectly upstage disease in some patients.

Despite an extensive literature documenting the sensitivity and specificity of PET/CT scanning, rarely publications exist to demonstrate an increased survival of patients with NSCLC due to the use of PET scanning. The studies of Fontaine et al. [49] showed that the introduction of routine PET scanning did not result in improved survival in the short or long term for patients undergoing resections for stage IA, IB and stage II, while a significant increased survival for stage III primary lung cancer $(P=0.03)$. They concluded that patients with stage III non-small-cell lung cancer should undergo PET scanning prior to surgical resection.

While in the study of Fischer et al. [46] 98 patients were assigned to PET/CT group, 91 patients were assigned 
to the conventional-staging group. They found that there were no significant differences in survival between the two groups; median survival was 31 months in the PET/CT group and 49 months in the conventional-staging group $(P=0.29)$. Therefore, they reported that the use of PET/CT for preoperative staging of NSCLC did not improve overall survival rate.

\section{The Cost-Effectiveness of PET/CT in NSCLC Staging}

A diagnostic effectiveness of PET/CT for staging NSCLC and superiority to conventional cancer imaging modalities has been demonstrated by many studies $[18,20,50,51]$. However, whether this technology should be widely applied in the staging of NSCLC remains at debate for its high examination costs. In addition, this may be offset in part by improvements in staging accuracy and examination times [52]. In recent years, incremental cost-effectiveness ratios (ICER), quality-adjusted life year (QALY) et al., were used to evaluate healthy economic of this technology. Several costeffectiveness analyses have been published evaluating PET in lung cancer staging, though data specifically on PET/CT is absence.

In a randomized trial performed by van Tinteren et al. [47], 96 patients were randomly assigned conventional workup (CWU) and 92 conventional workup and PET (CWU + PET). In the CWU group, 39 (41\%) patients had futile thoracotomies, compared with $19(21 \%)$ in the CWU + PET group (relative reduction $51 \%, P=0.003$ ). The addition of PET to CWU prevented futile surgery in one out of five patients with suspected NSCLC. Despite the additional PET costs, the total costs were lower in the PET group, mainly due to a reduction in the number of futile operations and general hospital days especially intensive care days. The additional use of PET in the staging of patients with NSCLC is feasible and safe, and it saves costs from a clinical and an economic perspective.

A study performed in Canada showed that PET for staging NSCLC without CT coregistration, compared with CT alone, led to a cost savings of $\$ 1,455$ combined with an increase in life expectancy of 3.1d [53]. Recently, the diagnostic value and cost-effectiveness have also been approved by the German health care system [54].

In one study based on 172 NSCLC patients who underwent diagnostic, contrast-enhanced helical CT and integrated PET/CT, Schreyögg et al. [55] reported that the incremental cost-effectiveness ratios (ICER) per correctly staged patient were $\$ 3,508$ for PET/CT versus CT alone. The ICER per quality-adjusted life year (QALY) gained were $\$ 79,878$ for PET/CT versus CT alone, decreasing to $\$ 69,563$ assuming a reduced loss of utility (0.10 QALY) due to surgical morbidity. The study showed that costs for PET/CT are within the commonly accepted range for diagnostic tests, therapies, or staging from the payer's perspective.

Søgaard et al. [56] performed a randomized clinical trial in which 189 patients were allocated to conventional staging $(n=91)$ or conventional staging + PET/CT $(n=98)$ and followed for 1 year after which the numbers of futile thoracotomies in each group were monitored. They showed that implementation of PET/CT into the diagnostic algorithm of staging NSCLC is cost-effective. The ICER was calculated to be $19,314 €$, meaning that PET/CT is cost-effective if the provider's willingness to pay (WTP) is $50,000 €$ per avoided futile thoracotomy. Including comorbidity-related costs, we found that the cost-effectiveness of PET/CT for staging NSCLC depends on the WTP in order to avoid a futile thoracotomy for which there is no true threshold value. A futile thoracotomy can be futile either because it is performed in patients with a benign lung lesion or because it is performed in patients with unresectable, end-stage cancer.

\section{The Limitation of PET/CT in Staging of Non-Small-Cell Lung Cancer}

Although PET/CT is an accurate and noninvasive method in the staging of non-small-cell lung cancer, many pitfalls exist. Imprecise physiologic and anatomic registration, most common adjacent to the diaphragm and heart, can lead to misregistration artifact [57]. Misregistration occurs as a result of differences in the position of the patient during the CT and PET examination. This may occur either as a result of voluntary motion of the patient during the examination or more commonly because of discrepancies in the phases of the patient's respiratory cycle during the $\mathrm{CT}$ and PET examinations [52]. Misregistration can lead to incorrect anatomical registration of PET and CT images, which can affect the accurate localization of uptake within pulmonary masses or lymph nodes. Cohade et al. [58] reported the mean discrepancy between the location of pulmonary lesions on PET and CT was approximately $7 \mathrm{~mm}$, with better registration at the lung apices than bases. These misregistrations can make micro-lesions missed, resulting in false negative results.

As a general rule uptake of $S U V(\max ) \geq 2.5$ was considered to indicate a malignant lesion, and $\operatorname{SUV}(\max )<2.5$ was considered to indicate a benign lesion [59]. Many processes with increased metabolic activities show increased FDG uptake on PET/CT imaging. Several benign lesions that have increased glucose metabolism can accumulate FDG and can be misinterpreted as malignant, such as infection, inflammation, and infarct [10]. In addition, physiological uptake of FDG usually found in the brain, heart, gastrointestinal and genitourinary tracts, and striated muscles show increased FDG uptake on PET/CT imaging. Iatrogenic causes of focal or diffuse FDG uptake include, granulation tissue, healing wounds, chest tubes and gastrotomy tubes, percutaneous needle biopsy, and mediastinoscopy [60]. These increased FDG uptake can lead to false positive results. There is some evidence to suggest that "dual-time-point" imaging may be helpful in differentiating malignant versus inflammatory and infectious processes [61].

False-negative FDG uptake includes technological limitations of PET/CT and inherent properties of neoplasm. Lesions that measure less than two to three times than the spatial resolution of the scanner will appear less active 
and a lower SUV due to the partial volume effect. Lesions containing little cells, low metabolic neoplasm, and highly differentiated tumors can lead to false negative results. It is important for us to know these potential pitfalls in order to get more accuracy staging of non-small-cell lung cancer.

\section{Conclusion}

The accurate staging of NSCLC is important in determining optimal treatment strategy and getting a better prognosis. Integrated PET/CT combining the benefits of PET and CT and minimizing their limitations is a potential tool in the staging of non-small-cell lung cancer. It reduces futile treatment and its associated morbidity and cost, improving the quality of life and being cost-effectiveness.

\section{Abbreviations}

$\begin{array}{ll}\text { PET/CT: } & \begin{array}{l}\text { Positron emission tomography/computed } \\ \text { tomography }\end{array} \\ \text { NSCLC: } & \text { Non-small-cell lung cancer } \\ \text { CT: } & \text { Computed tomography } \\ \text { PET: } & \text { Positron emission tomography } \\ { }^{18} \text { F-FDG: } & { }^{18} \text { F-fluorodeoxyglucose } \\ \text { MRI: } & \text { Magnetic resonance imaging } \\ \text { EBUS: } & \text { Endoscopic bronchial ultrasound } \\ { }^{99 m} \text { Tc-MDP: } & { }^{99 m} \text { Tc-methylene diphosphonate } \\ \text { SUV: } & \text { Standardized uptake value } \\ \text { FLT: } & { }^{18} \text { F-fluorothymidine } \\ \text { ICER: } & \text { Incremental cost-effectiveness ratios } \\ \text { QALY: } & \text { Quality-adjusted life year } \\ \text { CWU: } & \text { Conventional workup } \\ \text { CWU + PET: } & \text { Conventional workup and PET } \\ \text { WTP: } & \text { Willingness to pay. }\end{array}$

\section{Acknowledgments}

This work is partly sponsored by grants from the Zhejiang Provincial Natural Science Foundation of China (Z2110230), Health Bureau of Zhejiang Province (2010ZA075), National Science Foundation of China (NSFC) (no. 81101023, 81170306,81173468 ), and Ministry of Science and Technology of China (2011CB504400).

\section{References}

[1] D. Morgensztern, B. Goodgame, M. Q. Baggstrom, F. Gao, and R. Govindan, "The effect of FDG-PET on the stage distribution of non-small cell lung cancer," Journal of Thoracic Oncology, vol. 3, no. 2, pp. 135-139, 2008.

[2] A. Jemal, R. Siegel, E. Ward et al., "Cancer statistics, 2008," CA: A Cancer Journal for Clinicians, vol. 58, no. 2, pp. 71-96, 2008.

[3] H. C. Steinert, "PET and PET-CT of lung cancer," Methods in Molecular Biology, vol. 727, pp. 33-51, 2011.

[4] W. De Wever, J. Coolen, and J. A. Verschakelen, "Integrated PET/CT and cancer imaging," JBR-BTR, vol. 92, no. 1, pp. 1319, 2009.

[5] S. Y. Low, P. Eng, G. H. W. Keng, and D. C. E. Ng, "Positron emission tomography with CT in the evaluation of non-small cell lung cancer in populations with a high prevalence of tuberculosis," Respirology, vol. 11, no. 1, pp. 84-89, 2006.

[6] P. McQuade, D. J. Rowland, J. S. Lewis, and M. J. Welch, "Positron-emitting isotopes produced on biomedical cyclotrons," Current Medicinal Chemistry, vol. 12, no. 7, pp. 807-818, 2005.

[7] W. V. Vogel, W. J. Oyen, J. O. Barentsz, J. H. Kaanders, and F. H. Corstens, "PET/CT: panacea, redundancy, or something in between?" Journal of Nuclear Medicine, vol. 45, supplement 1, pp. 15S-24S, 2004.

[8] Y. Y. Yau, W. S. Chan, Y. M. Tam et al., "Application of intravenous contrast in PET/CT: does it really introduce significant attenuation correction error?" Journal of Nuclear Medicine, vol. 46, no. 2, pp. 283-291, 2005.

[9] S. Kligerman and S. Digumarthy, "Staging of non-small cell lung cancer using integrated PET/CT," American Journal of Roentgenology, vol. 193, no. 5, pp. 1203-1211, 2009.

[10] S. Tsim, C. A. O’Dowd, R. Milroy, and S. Davidson, "Staging of non-small cell lung cancer (NSCLC): a review," Respiratory Medicine, vol. 104, no. 12, pp. 1767-1774, 2010.

[11] R. Rami-Porta, J. J. Crowley, and P. Goldstraw, "The revised TNM staging system for lung cancer," Annals of Thoracic and Cardiovascular Surgery, vol. 15, no. 1, pp. 4-9, 2009.

[12] L. L. Carr, J. H. Finigan, and J. A. Kern, "Evaluation and treatment of patients with non-small cell lung cancer," Medical Clinics of North America, vol. 95, no. 6, pp. 1041-1054, 2011.

[13] D. S. Ettinger, W. Akerley, G. Bepler et al., "Non-small cell lung cancer: clinical practice guidelines in oncology," Journal of the National Comprehensive Cancer Network, vol. 8, no. 7, pp. 740$801,2010$.

[14] J. P. C. Grutters, A. G. H. Kessels, M. Pijls-Johannesma, D. De Ruysscher, M. A. Joore, and P. Lambin, "Comparison of the effectiveness of radiotherapy with photons, protons and carbon-ions for non-small cell lung cancer: a meta-analysis," Radiotherapy and Oncology, vol. 95, no. 1, pp. 32-40, 2010.

[15] R. Timmerman, R. Paulus, J. Galvin et al., "Stereotactic body radiation therapy for inoperable early stage lung cancer," JAMA, vol. 303, no. 11, pp. 1070-1076, 2010.

[16] D. Lardinois, W. Weder, T. F. Hany et al., "Staging of non-small-cell lung cancer with integrated positron-emission tomography and computed tomography," The New England Journal of Medicine, vol. 348, no. 25, pp. 2500-2507, 2003.

[17] B. M. Fischer and J. Mortensen, "The future in diagnosis and staging of lung cancer: positron emission tomography," Respiration, vol. 73, no. 3, pp. 267-276, 2006.

[18] W. Wever, S. Ceyssens, L. Mortelmans et al., "Additional value of PET-CT in the staging of lung cancer: comparison with CT alone, PET alone and visual correlation of PET and CT," European Radiology, vol. 17, no. 1, pp. 23-32, 2007.

[19] W. R. Webb, C. Gatsonis, E. A. Zerhouni et al., "CT and MR imaging in staging non-small cell bronchogenic carcinoma: report of the Radiologic Diagnostic Oncology Group," Radiology, vol. 178, no. 3, pp. 705-713, 1991.

[20] S. S. Shim, K. S. Lee, B. T. Kim et al., "Non-small cell lung cancer: prospective comparison of integrated FDG PET/CT and CT alone for preoperative staging," Radiology, vol. 236, no. 3, pp. 1011-1019, 2005.

[21] S. Pauls, A. K. Buck, K. Hohl et al., "Improved non-invasive TStaging in non-small cell lung cancer by integrated 18F-FDG PET/CT," NuklearMedizin, vol. 46, no. 1, pp. 9-14, 2007.

[22] J. A. Verschakelen, W. De Wever, and J. Bogaert, "Role of computed tomography in lung cancer staging," Current Opinion in Pulmonary Medicine, vol. 10, no. 4, pp. 248-255, 2004. 
[23] K. M. Kerr, D. Lamb, C. G. Wathen, W. S. Walker, and N. J. Douglas, "Pathological assessment of mediastinal lymph nodes in lung cancer: implications for non-invasive mediastinal staging," Thorax, vol. 47, no. 5, pp. 337-341, 1992.

[24] N. C. Gupta, W. J. Tamim, G. G. Graeber, H. A. Bishop, and G. R. Hobbs, "Mediastinal lymph node sampling following positron emission tomography with fluorodeoxyglucose imaging in lung cancer staging," Chest, vol. 120, no. 2, pp. 521$527,2001$.

[25] G. E. Darling, D. E. Maziak, R. I. Inculet et al., "Positron emission tomography-computed tomography compared with invasive mediastinal staging in non-small cell lung cancer: results of mediastinal staging in the early lung positron emission tomography trial," Journal of Thoracic Oncology, vol. 6, no. 8, pp. 1367-1372, 2011.

[26] T. Y. Jeon, K. S. Lee, C. A. Yi et al., "Incremental value of PET/CT over CT for mediastinal nodal staging of nonsmall cell lung cancer: comparison between patients with and without idiopathic pulmonary fibrosis," American Journal of Roentgenology, vol. 195, no. 2, pp. 370-376, 2010.

[27] A. Billé, E. Pelosi, A. Skanjeti et al., "Preoperative intrathoracic lymph node staging in patients with non-small-cell lung cancer: accuracy of integrated positron emission tomography and computed tomography," European Journal of CardioThoracic Surgery, vol. 36, no. 3, pp. 440-445, 2009.

[28] B.-J. Liu, J.-C. Dong, C.-Q. Xu et al., "Accuracy of 18FFDG PET/CT for lymph node staging in non-small-cell lung cancers," Chinese Medical Journal, vol. 122, no. 15, pp. 17491754, 2009.

[29] W. F. Yang, G. Z. Tan, Z. Fu, and J. M. Yu, "[Evaluation of the diagnostic value of (18)F-FDG PET-CT and enhanced CT for staging of lymph node metastasis in non-small cell lung cancer]," Chinese Journal of Oncology, vol. 31, no. 12, pp. 925928, 2009.

[30] K. G. Tournoy, S. Maddens, R. Gosselin, G. Van Maele, J. P. Van Meerbeeck, and A. Kelles, "Integrated FDG-PET/CT does not make invasive staging of the intrathoracic lymph nodes in non-small cell lung cancer redundant: a prospective study," Thorax, vol. 62, no. 8, pp. 696-701, 2007.

[31] C. A. Yi, K. S. Lee, B. T. Kim et al., "Efficacy of helical dynamic CT versus integrated PET/CT for detection of mediastinal nodal metastasis in non-small cell lung cancer," American Journal of Roentgenology, vol. 188, no. 2, pp. 318-325, 2007.

[32] C. Perigaud, B. Bridji, J. C. Roussel et al., "Prospective preoperative mediastinal lymph node staging by integrated positron emission tomography-computerised tomography in patients with non-small-cell lung cancer," European Journal of Cardio-Thoracic Surgery, vol. 36, no. 4, pp. 731-736, 2009.

[33] M. Metin, N. Citak, A. Sayar et al., "The role of extended cervical mediastinoscopy in staging of non-small cell lung cancer of the left lung and a comparison with integrated positron emission tomography and computed tomography: does integrated positron emission tomography and computed tomography reduce the need for invasive procedures?" Journal of Thoracic Oncology, vol. 6, no. 10, pp. 1713-1719, 2011.

[34] L. E. Quint, S. Tummala, L. J. Brisson et al., "Distribution of distant metastases from newly diagnosed non-small cell lung cancer," Annals of Thoracic Surgery, vol. 62, no. 1, pp. 246-250, 1996.

[35] M. T. Truong, C. Viswanathan, and J. J. Erasmus, "Positron emission tomography/computed tomography in lung cancer staging, prognosis, and assessment of therapeutic response," Journal of Thoracic Imaging, vol. 26, no. 2, pp. 132-146, 2011.
[36] A. V. Taira, R. J. Herfkens, S. S. Gambhir, and A. Quon, "Detection of bone metastases: assessment of integrated FDG PET/CT imaging," Radiology, vol. 243, no. 1, pp. 204-211, 2007.

[37] G. Heinz-Peer, S. Hönigschnabl, B. Schneider, B. Niederle, K. Kaserer, and G. Lechner, "Characterization of adrenal masses using MR imaging with histopathologic correlation," American Journal of Roentgenology, vol. 173, no. 1, pp. 15-22, 1999.

[38] S. Jana, T. Zhang, D. M. Milstein, C. R. Isasi, and M. D. Blaufox, "FDG-PET and CT characterization of adrenal lesions in cancer patients," European Journal of Nuclear Medicine and Molecular Imaging, vol. 33, no. 1, pp. 29-35, 2006.

[39] M. S. Yon, S. L. Kyung, B. T. Kim et al., “18F-FDG PET versus 18F-FDG PET/CT for adrenal gland lesion characterization: a comparison of diagnostic efficacy in lung cancer patients," Korean Journal of Radiology, vol. 9, no. 1, pp. 19-28, 2008.

[40] C. Ansquer, S. Scigliano, E. Mirallié et al., "18F-FDG PET/CT in the characterization and surgical decision concerning adrenal masses: a prospective multicentre evaluation," European Journal of Nuclear Medicine and Molecular Imaging, vol. 37, no. 9, pp. 1669-1678, 2010.

[41] M. Yun, W. Kim, N. Alnafisi, L. Lacorte, S. Jang, and A. Alavi, "18F-FDG PET in characterizing adrenal lesions detected on CT or MRI," Journal of Nuclear Medicine, vol. 42, no. 12, pp. 1795-1799, 2001.

[42] M. Perri, P. Erba, D. Volterrani et al., "Adrenal masses in patients with cancer: PET/CT characterization with combined CT histogram and standardized uptake value PET analysis," American Journal of Roentgenology, vol. 197, no. 1, pp. 209216, 2011.

[43] S. Chong, S. L. Kyung, Y. K. Ha et al., "Integrated PETCT for the characterization of adrenal gland lesions in cancer patients: diagnostic efficacy and interpretation pitfalls," Radiographics, vol. 26, no. 6, pp. 1811-1824, 2006.

[44] P. Goldstraw, J. Crowley, K. Chansky et al., "The IASLC lung cancer staging project: proposals for the revision of the TNM stage groupings in the forthcoming (seventh) edition of the TNM classification of malignant tumours," Journal of Thoracic Oncology, vol. 2, no. 8, pp. 706-714, 2007.

[45] A. Salskov, V. S. Tammisetti, J. Grierson, and H. Vesselle, "FLT: measuring Tumor Cell Proliferation In Vivo With Positron Emission Tomography and 3'-Deoxy-3'$\left[{ }^{18} \mathrm{~F}\right]$ Fluorothymidine," Seminars in Nuclear Medicine, vol. 37, no. 6, pp. 429-439, 2007.

[46] B. Fischer, U. Lassen, J. Mortensen et al., "Preoperative staging of lung cancer with combined PET-CT," The New England Journal of Medicine, vol. 361, no. 1, pp. 32-39, 2009.

[47] H. van Tinteren, O. S. Hoekstra, E. F. Smit et al., "Effectiveness of positron emission tomography in the preoperative assessment of patients with suspected non-small-cell lung cancer: the PLUS multicentre randomised trial," The Lancet, vol. 359, no. 9315, pp. 1388-1392, 2002.

[48] R. C. Viney, M. J. Boyer, M. T. King et al., "Randomized controlled trial of the role of positron emission tomography in the management of stage I and II non-small-cell lung cancer," Journal of Clinical Oncology, vol. 22, no. 12, pp. 2357-2362, 2004.

[49] E. Fontaine, J. McShane, M. Carr et al., "Does positron emission tomography scanning improve survival in patients undergoing potentially curative lung resections for non-smallcell lung cancer?" European Journal of Cardio-Thoracic Surgery, vol. 40, no. 3, pp. 642-646, 2011. 
[50] A. K. Buck, K. Herrmann, T. Stargardt, T. Dechow, B. J. Krause, and J. Schreyögg, "Economic evaluation of PET and PET/CT in oncology: evidence and methodologic approaches," Journal of Nuclear Medicine Technology, vol. 38, no. 1, pp. 6-17, 2010.

[51] T. L. Allen, A. T. K. Kendi, M. O. Mitiek, and M. A. Maddaus, "Combined contrast-enhanced computed tomography and 18-fluoro-2-deoxy-D-glucose-positron emission tomography in the diagnosis and staging of non-small cell lung cancer," Seminars in Thoracic and Cardiovascular Surgery, vol. 23, no. 1, pp. 43-50, 2011.

[52] A. Devaraj, G. J. R. Cook, and D. M. Hansell, "PET/CT in non-small cell lung cancer staging-promises and problems," Clinical Radiology, vol. 62, no. 2, pp. 97-108, 2007.

[53] J. S. Sloka, P. D. Hollett, and M. Mathews, "Cost-effectiveness of positron emission tomography for non-small cell lung carcinoma in Canada," Medical Science Monitor, vol. 10, no. 5, pp. MT73-MT80, 2004.

[54] A. K. Buck, K. Herrmann, and J. Schreyögg, "PET/CT for staging lung cancer: costly or cost-saving?” European Journal of Nuclear Medicine and Molecular Imaging, vol. 38, no. 5, pp. 799-801, 2011.

[55] J. Schreyögg, J. Weller, T. Stargardt et al., "Cost-effectiveness of hybrid PET/CT for staging of non-small cell lung cancer," Journal of Nuclear Medicine, vol. 51, no. 11, pp. 1668-1675, 2010.

[56] R. Søgaard, B. M. B. Fischer, J. Mortensen, L. Højgaard, and U. Lassen, "Preoperative staging of lung cancer with PET/CT: cost-effectiveness evaluation alongside a randomized controlled trial," European Journal of Nuclear Medicine and Molecular Imaging, vol. 38, no. 5, pp. 802-809, 2011.

[57] M. D. Gilman, A. J. Fischman, V. Krishnasetty, E. F. Halpern, and S. L. Aquino, "Optimal CT breathing protocol for combined thoracic PET/CT," American Journal of Roentgenology, vol. 187, no. 5, pp. 1357-1360, 2006.

[58] C. Cohade, M. Osman, L. T. Marshall, and R. L. Wahl, "PETCT: accuracy of PET and CT spatial registration of lung lesions," European Journal of Nuclear Medicine and Molecular Imaging, vol. 30, no. 5, pp. 721-726, 2003.

[59] M. Okada, T. Shimono, Y. Komeya et al., "Adrenal masses: the value of additional fluorodeoxyglucose-positron emission tomography/computed tomography (FDG-PET/CT) in differentiating between benign and malignant lesions," Annals of Nuclear Medicine, vol. 23, no. 4, pp. 349-354, 2009.

[60] T. F. Hany, J. Heuberger, and G. K. von Schulthess, "Iatrogenic FDG foci in the lungs: a pitfall of PET image interpretation," European Radiology, vol. 13, no. 9, pp. 2122-2127, 2003.

[61] S.-J. Kim, Y.-K. Kim, I. J. Kim, Y. D. Kim, and M. K. Lee, "Limited predictive value of dual-time-point F-18 FDG PET/CT for evaluation of pathologic N1 status in NSCLC patients," Clinical Nuclear Medicine, vol. 36, no. 6, pp. 434439, 2011. 


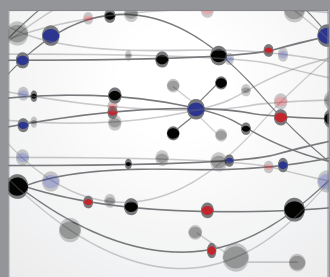

The Scientific World Journal
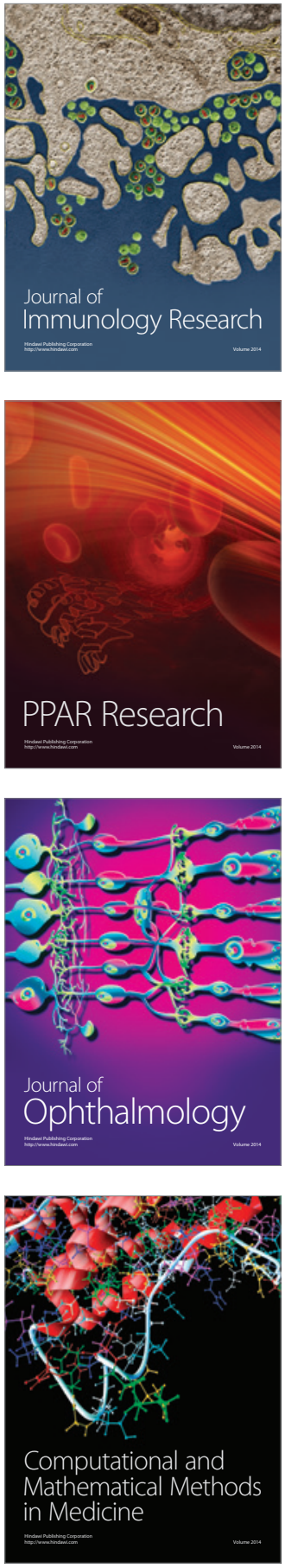

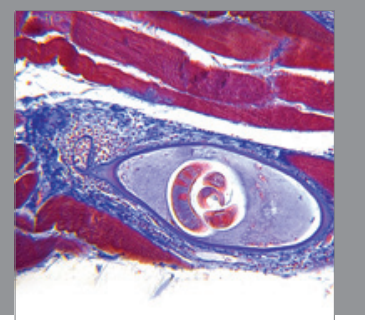

Gastroenterology

Research and Practice
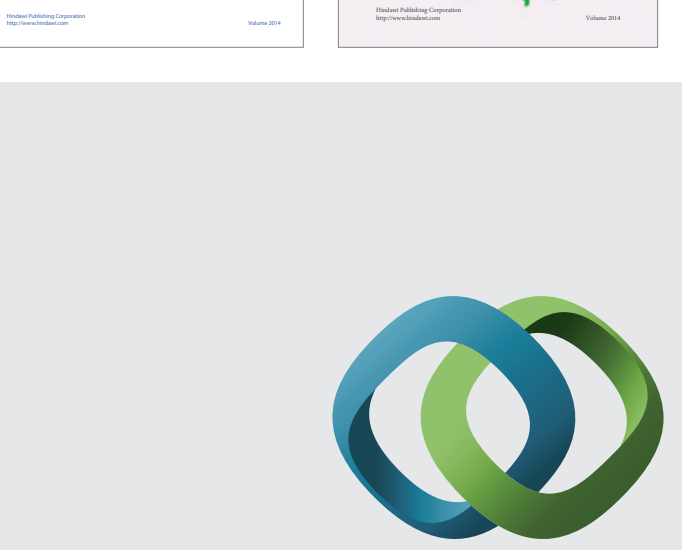

\section{Hindawi}

Submit your manuscripts at

http://www.hindawi.com
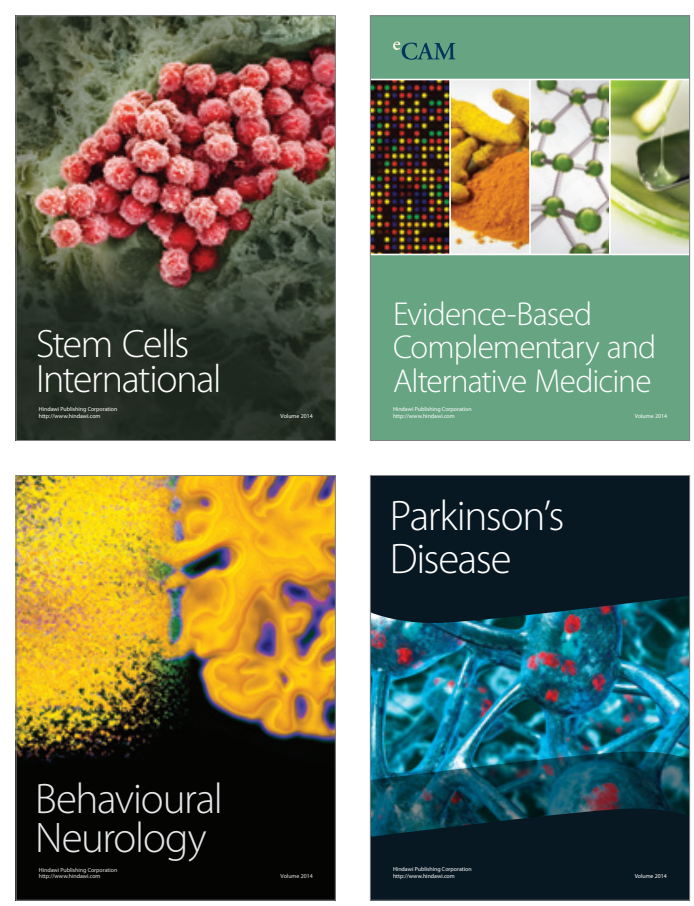

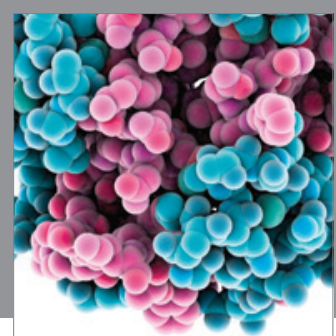

Journal of
Diabetes Research

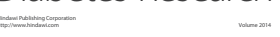

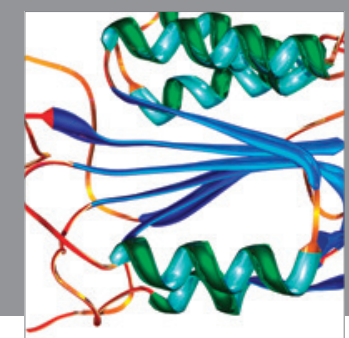

Disease Markers
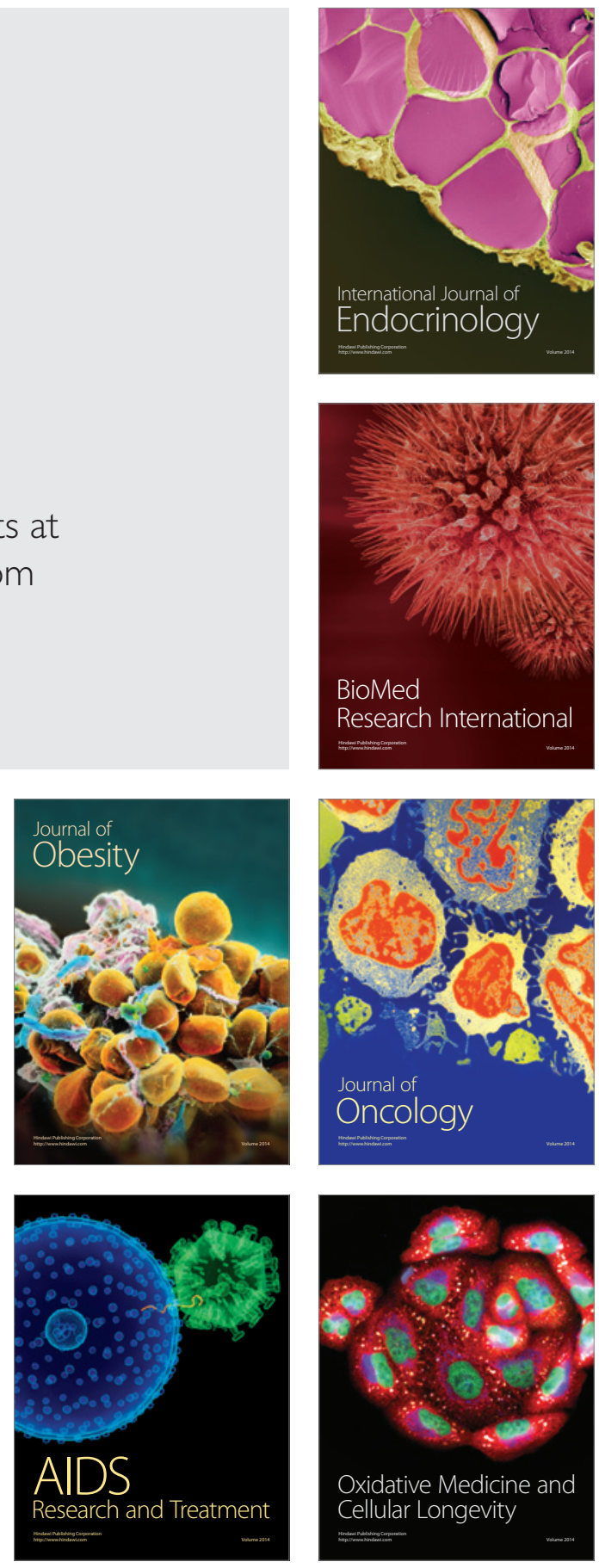